\title{
Nitrogen solubility in bridgmanite under lower-mantle conditions
}

\author{
K. FUKUYAMA ${ }^{*}{ }^{1}$, H. KAGI ${ }^{1}$, T. INOUE ${ }^{2,3}$, S. KAKIZAWA ${ }^{1}$, \\ T. SHINMEI ${ }^{3}$, Y. SANO ${ }^{4,5}$, C. DELIGNY ${ }^{6}$, E. FÜRI $^{6}$ \\ ${ }^{1}$ Graduate School of Science, The University of Tokyo, \\ Tokyo 113-0033, Japan \\ (ko.fukuyama@eqchem.s.utokyo.ac.jp) \\ ${ }^{2}$ Graduate School of Science, Hiroshima University \\ ${ }^{3}$ Geodynamic Research Center, Ehime Univerisity \\ ${ }^{4}$ Atmosphere and Ocean Research Institute, The University \\ of Tokyo \\ ${ }^{5}$ Institute of Surface-Earth System Science, Tianjin \\ University \\ ${ }^{6}$ Centre de Recherches Pétrographiques et Géochimiques
}

Nitrogen occupies the most of the Earth's atmosphere and is one of essential elements of life. Despite its importance, nitrogen behavior in the Earth's interior remains unclear. Nitrogen in the bulk silicate Earth is more depleted than other volatiles (Marty et al., 2012). Experimental researches suggested that nitrogen has been stored in a deep mantle through magma ocean (Li et al., 2013; Yoshioka et al., 2018). Yoshioka et al. (2018) is the only study reporting a nitrogen solubility in bridgmanite which occupies about $80 \mathrm{vol} \%$ of a lower mantle, but their experimental condition was limited at $24 \mathrm{GPa}$ and $1600{ }^{\circ} \mathrm{C}$.

In this research, we investigated temperature dependence on nitrogen solubility in bridgmanite. High-pressure experiments were conducted using multi-anvil apparatus installed at GRC, Ehime University. The pressure condition was $28 \mathrm{GPa}$ and temperature conditions ranged from $1400{ }^{\circ} \mathrm{C}$ to $1700{ }^{\circ} \mathrm{C}$. Fe-FeO buffer was used to control the redox state equal to that of the lower mantle. Nitrogen in recovered samples was analyzed using a secondary ionization mass spectrometer (HR-1280, Cameca) installed at CRPG, France.

A series of experimental results revealed that nitrogen solubility in bridgmanite increased from 1.8 to $5.6 \mathrm{ppm}$ with increasing temperature. The results implies that the lower mantle can store 3-4 PAN (PAN: mass of Present Atmospheric Nitrogen) only by bridgmanite at maximum. Bridgmanite firstly crystallized in cooling magma ocean (e.g. Ozawa et al., 2018) and would capture nitrogen there. Our study suggests that bridgmanite played an important role in reserving nitrogen through solidification of magma ocean. This formation of "hidden" nitrogen reservoir in the lower mantle may constrain atmosphere-mantle coevolution and deplete nitrogen compared to other volatiles in the Earth where life has been confirmed in the solar system. 